\title{
Prevalence of Helicobacter pylori infection and related gastroduodenal lesions in spouses of Helicobacter pylori positive patients with duodenal ulcer
}

\author{
F Parente, G Maconi, O Sangaletti, M Minguzzi, L Vago, E Rossi, G Bianchi Porro
}

\begin{abstract}
Background-To date, very few studies have evaluated the risk of infection among spouses of Helicobacter pylori positive patients and their results are conflicting. Aim-To assess the seroprevalence of $H$ pylori infection in spouses of $\mathrm{H}$ pylori positive patients with duodenal ulcer as compared with age and sex matched volunteer blood donors, as well as the frequency of endoscopic gastroduodenal lesions in these spouses, according to the presence or absence of gastrointestinal complaints. Methods-Some 124 spouses (48\% males) of patients with duodenal ulcer consecutively seen over a 10 month period were studied. They were all screened for serum IgG anti-H pylori antibodies and asked to complete a questionnaire with particular reference to the presence of chronic or recurrent dyspepsia. Upper gastrointestinal tract endoscopy with antral and corpus biopsy specimens taken for histological examination and urease rapid test was offered to all seropositive spouses. Volunteer blood donors (248), living in Milan and matched for age, sex, north-south origins, and socioeconomic status to the cases, were used as controls. Results-Spouses of patients with duodenal ulcer had a significantly higher seroprevalence of $\boldsymbol{H}$ pylori infection than controls $(71 \% \quad v 58 \%, p<0.05) ; 30$ of 88 $(34 \%) H$ pylori positive spouses complained of dyspeptic symptoms compared with only four of $34(12 \%)$ seronegative spouses $(p<0 \cdot 02)$. At endoscopy, $H$ pylori infection was confirmed in 48 of 49 $(98 \%)$ seropositive spouses. The endoscopic findings in those spouses showed active duodenal ulcer in eight $(17 \%)$, duodenal scar and cap deformity in two $(4 \%)$, active gastric ulcer in two $(4 \%)$, erosive duodenitis in three $(6 \%)$, antral erosions in two (4\%), antral erosions plus duodenitis in one, and peptic oesophagitis in another patient. The prevalence of major endoscopic lesions was significantly higher in symptomatic spouses than in those who had never been symptomatic. Conclusions-These findings show that being the spouse of an $H$ pylori positive patient with duodenal ulcer may increase the risk of $H$ pylori colonisation and perhaps of peptic ulcer disease, and raises
\end{abstract}

questions as to whether serological screening of cohabiting partners of $H$ pylori positive patients with duodenal ulcer may be indicated.

(Gut 1996; 39: 629-633)

Keywords: $H$ pylori infection, duodenal ulcer, spouses.

Although the source and mode of transmission of Helicobacter pylori remain uncertain, recent studies have reported clustering of $H$ pylori organisms among family members. ${ }^{1-4}$ This may reflect either the importance of a genetic influence for susceptibility to $H$ pylori or a community acquired, such as person to person, mode of transmission.

Another way to prove the importance of normal household contacts in the acquisition of $H$ pylori is to evaluate the risk of infection among spouses of $H$ pylori positive patients compared with the general population. This cannot involve a genetic predisposition, unlike children of infected patients. So far, only a few studies have been published on this topic and their results are conflicting. ${ }^{35-7}$ Furthermore, most of these studies have included patients with different gastroduodenal pathologies (peptic ulcer, non-ulcer dyspepsia and asymptomatic gastritis), each of which might have occurred with different strains of $\mathrm{H}$ pylori, ${ }^{8}$ so their results are difficult to interpret.

Up to now, the clinical relevance of seropositivity in spouses of $H$ pylori positive patients remains unknown because no study has evaluated the prevalence and spectrum of $H$ pylori related gastroduodenal lesions in seropositive spouses according to the presence or absence of gastrointestinal symptoms.

This study was designed (a) to estimate the prevalence of $H$ pylori infection among spouses of $H$ pylori positive patients and to compare this with a matched group of volunteer blood donors, and (b) to evaluate the endoscopic and histological findings of those spouses according to their gastrointestinal symptoms.

\section{Methods}

\section{STUDY POPULATION}

Cases

Over a period of 10 months (September 1993June 1994) 168 consecutive patients with 
active duodenal ulcer, diagnosed by an upper gastrointestinal tract (GI) endoscopy at L Sacco Hospital, were found to have an $H$ pylori infection, determined by identification of the organism by histological examination and a positive urease test. Of these patients, 134 had a cohabiting spouse and were therefore considered eligible for the study. A spouse was defined as a husband or wife who had been living in the patient's household for at least four years. Enrolment criteria also required that both partners had been born in Italy and had never lived abroad during their childhood. There were 124 spouses (64 women) aged $27-74$ (mean 48) years that met these criteria and gave their informed consent to participate in the study.

Each partner completed a standard questionnaire separately, which was reviewed by a trained interviewer (FP or GM) who was unaware of his/her $H$ pylori status. The questionnaire sought information about: (1) demographic factors such as age, race, sex, place of birth in Italy, educational level, occupation, number of children; (2) medical history, with particular reference to an already documented upper GI pathology or to a history of chronic or recurrent foregut symptoms (see below); (3) consumption of drugs in the past, especially antacids, $\mathrm{H}_{2}$ blockers, proton pump inhibitors, bismuth salts or antibiotics for periods longer than two weeks, and during the last three months. Each subject was also questioned about tobacco and alcohol use and whether they kept pets at home.

In particular, the evaluation of upper GI symptoms consisted of a questionnaire including the presence and severity of six dyspeptic symptoms: diurnal epigastric pain, nocturnal epigastric pain, nausea and vomiting, heartburn, anorexia and early satiety, regurgitation. These symptoms were scored for severity from 0 to 3, where $0=$ absent, $1=$ present but not interfering with normal daily activity, $2=$ interfering with daily work, $3=$ severe. A spouse was defined as being 'symptomatic' if they had repeatedly experienced dyspeptic symptoms in the past or had been having dyspeptic symptoms for more than three weeks.

A serum sample was obtained from each spouse who took part in the study for assay of IgG antibody to $H$ pylori specific antigens (see below).

\section{Controls}

Serum samples collected from blood units of volunteer blood donors living in Milan and attending the Blood Transfusional Centre of L Sacco Hospital served as the control group representative of the general population. In Italy, blood donors are screened and anyone taking regular non-steroidal anti-inflammatory drugs (NSAIDs), corticosteroids, antibiotics, immunosuppressants or any other cytotoxic agent or who is found to have a biochemical abnormality is not allowed to donate blood. In contrast, a history of peptic ulcer disease is not considered an exclusion criterion for giving blood. Men are allowed to donate blood every three months and women every six months. Sex, age, place of birth in Italy, educational level, and occupation of donors were provided; in particular, because previous studies showed a close link between socioeconomic status and educational level, ${ }^{9}$ we used the latter parameter to determine the socioeconomic status of cases and controls; three different degrees of educational level were distinguished: high (university or high school), medium (secondary school education) and low (primary school only). According to their place of birth in Italy, cases and controls were classified into four different areas of origin: (a) northern urban area, (b) northern rural area, (c) southern urban area, and (d) southern rural area. Each spouse was then matched by sex, age range ( \pm five years), area of origin in Italy, and socioeconomic status (that is the educational level) with two controls. Serum samples were excluded from this comparison group if there was gross haemolysis of the specimen.

\section{METHODS}

Serum samples from cases and controls were screened for anti- $H$ pylori IgG by enzyme linked immunosorbent assay (ELISA) using a commercial kit (Eurospital Helori test). The results were expressed as an index percentage obtained by comparing the patient value with the positive control. More than $19 \%$ was defined as a positive result. This cut off value was determined in our laboratory by the analysis of 200 patients.

Each seropositive spouse was given the opportunity of undergoing an upper GI endoscopy, regardless of the presence or absence of previous or current dyspeptic symptoms. All the endoscopies were performed by the same investigator (FP), after intravenous premedication of the patient (diazepam); an Olympus videoendoscope GIF-100 was used.

An active duodenal or gastric ulcer was defined as a mucosal ulceration with a maximum diameter not less than $5 \mathrm{~mm}$. A healed duodenal ulcer was defined as a clearly visible mucosal scar in the context of a deformed duodenal cap. A diagnosis of antral erosion or erosive duodenitis was made in the presence of multiple discrete focal erosions of less than $5 \mathrm{~mm}$. Diffuse or focal erythema of gastric and duodenal mucosa was recorded, but not considered to be a mucosal lesion. Three antral biopsy specimens (from 2 to $5 \mathrm{~cm}$ from the pylorus) and three biopsy specimens from the corpus (along the greater curve) were taken in all subjects, four for histology (haematoxylin and eosin and Giemsa staining) and two for the urease rapid test (CP test, Gist-Brocades Farma, Milan, Italy). Gastric histopathological examination was evaluated without knowledge of the subject's $H$ pylori status and was classified according to the Sydney system. ${ }^{10}$ In particular, a detailed protocol was used to evaluate gastritis activity and total immune-inflammatory cells in the antrum and corpus using four different categories of severity for each parameter (absent, mild, moderate, and severe). ${ }^{11} \mathrm{H}$ pylori infection was diagnosed by identification of the organism by histological examination and by the rapid urease test. 
The study was approved by the L Sacco Hospital Ethical Committee and written consent for endoscopy was obtained from all subjects.

\section{STATISTICAL ANALYSIS}

Multiple logistic regression analysis was carried out to model the seroprevalence of $H$ pylori taking into account the two groups of subjects, the three classes of age $(20-34$ years $=0,35-50$ years $=1$ and $>50$ years $=2$ considered as a continuous variable for assessing the presence of a linear trend), and the interaction between them. Gastritis scores are reported as medians and range; statistical comparison between gastritis scores of symptomatic and 'neversymptomatic' spouses was made by the Wilcoxon's rank sum test.

\section{Results}

Eighty eight (71\%) of 124 spouses were found to be positive for antibodies to $H$ pylori (Table I) compared with 145 (58\%) of 248 specimens from age and sex matched controls $(p<0.05)$. The prevalence of high IgG titres progressively increased with age among controls, but not among spouses and was not correlated with sex. Indeed, within the youngest age strata (20-34 years), $73 \%$ of spouses were seropositive compared with $33 \%$ of blood donors, whereas in the oldest age strata ( $>50$ years) $75 \%$ of spouses were seropositive compared with $74 \%$ of blood donors (Table I); the rate of seropositivity was similar in males and females. The average duration of cohabitation within couples was $16 \cdot 8$ (range 4-39) years.

When interviewed, 30 (34\%) of 88 seropositive spouses gave a history of chronic or recurrent upper GI symptoms, in comparison with only four $(11 \%)$ of seronegative spouses

TABLE I Seroprevalence of $\mathrm{H}$ pylori infection by age strata

\begin{tabular}{llll}
\hline \multicolumn{4}{l}{ Seroprevalence } \\
\cline { 2 - 4 } Study group & $20-34$ & $35-50$ & $>50$ \\
year olds & year olds & year olds \\
\hline Spouses & $11 / 15(73)$ & $38 / 57(67)$ & $39 / 52(75)$ \\
Blood donors & $10 / 30(33)$ & $58 / 114(51)$ & $77 / 104(74)$ \\
\hline
\end{tabular}

Values in parentheses are percentages.

Overall, seroprevalence in blood donors was significantly different from that in spouses.

TABLE II Endoscopic findings in $\mathrm{H}$ pylori positive spouses according to the presence or absence of upper GI symptoms

\begin{tabular}{lclc}
\hline Symptomatic spouses & No & Never symptomatic spouses & No \\
\hline Active duodenal ulcer & $8(31)$ & Active gastric ulcer & $1(5)$ \\
Scar+cap deformity & $2(8)$ & Antral erosions & $1(5)$ \\
Erosive duodenitis & $2(8)$ & Erosive duodenitis & $1(5)$ \\
Active gastric ulcer & $1(4)$ & & \\
Antral erosions & $1(4)$ & & \\
Antral erosions+erosive duodenitis & $1(4)$ & & $19(86)$ \\
Peptic oesophagitis & $1(4)$ & Normal findings & 22 \\
Normal findings & $10(38)$ & Total & \\
Total & 26 & &
\end{tabular}

Values in parentheses are percentages.

For the percentage of endoscopic lesions in symptomatic $v$ never symptomatic spouses, $p=0.002$ ( $\chi^{2}$ test with Yates's correction). $(p<0.01)$. In particular, four seropositive spouses had a history of duodenal ulcer disease (three cases ascertained by endoscopy and in one case by barium meal), while another 14 spouses had been investigated for dyspepsia in the past by their general practitioner ( 12 by barium meal, two by upper GI endoscopy). In addition, three of the four seronegative spouses complaining of dyspepsia had been investigated previously (two by upper GI endoscopy, one by barium meal) with negative results. None of either the symptomatic or asymptomatic spouses had been previously tested for $H$ pylori infection, had received anti- $H$ pylori therapy, or had been taking NSAIDs regularly.

Forty nine $(56 \%)$ of 88 seropositive spouses agreed to undergo upper GI endoscopy with multiple biopsies of the antrum and gastric body; 26 had a history of upper GI symptoms, although only seven complained of dyspepsia at the time of endoscopy; the remaining 23 spouses had never complained of chronic or recurrent dyspepsia.

At endoscopy, $H$ pylori infection was confirmed in 48 of 49 (98\%) seropositive spouses by both Giemsa staining and the urease rapid test, whereas one subject had no evidence of $H$ pylori. On repeated serology her titre was similar to the previous level so that she was considered a false positive.

The endoscopic findings of those 48 patients with confirmed chronic $H$ pylori infection showed active duodenal ulcer in eight $(17 \%)$, duodenal scar and cap deformity in two (4\%), erosive duodenitis in three $(6 \%)$, active gastric ulcer in two (4\%), antral erosions in two (4\%), erosive duodenitis in three $(6 \%)$, active gastric ulcer in two (4\%), antral erosions in two (4\%), antral erosions plus duodenitis in one, and peptic oesophagitis in another, whereas no endoscopic lesions were documented in the remaining $29(60 \%)$. However, the prevalence of major gastroduodenal lesions was significantly higher in symptomatic than in asymptomatic $H$ pylori positive spouses (Table II).

In all the 48 spouses with evidence of $H$ pylori infection, histopathology showed antral gastritis, which was mild in $41 \%$, moderate in $45 \%$, and severe in $14 \%$. No significant difference was found in antral gastritis scores between symptomatic and asymptomatic spouses (Figure). In contrast, the only spouse who underwent endoscopy with no evidence of $H$ pylori infection also had a normal antral histology.

\section{Discussion}

Although the exact mode of transmission of $H$ pylori infection is unknown, numerous studies have indicated that a close human contact is related to a high prevalence of $H$ pylori infection. Indeed, studies of institutionalised people, ${ }^{12}$ gastroenterologists, ${ }^{13}$ nurses, ${ }^{14}$ and familial clustering ${ }^{13}$ have reported an increased prevalence of $H$ pylori antibodies, thus suggesting a direct person to person spread of the infection.

A study of transmission within couples is another way to examine the risk of acquisition 


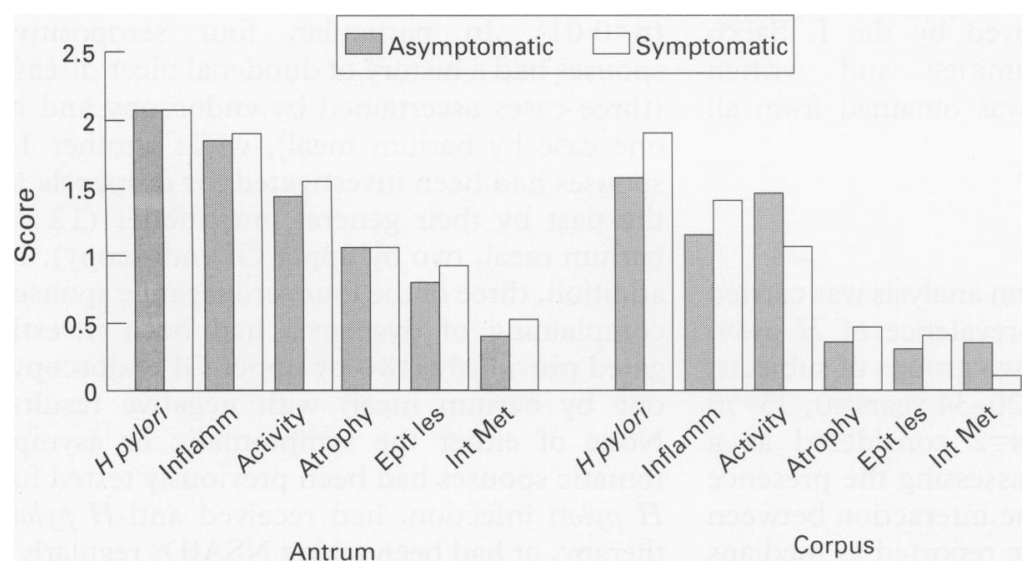

Gastritis scores (antrum and corpus) in asymptomatic and symptomatic spouses. No significant difference was found in $\mathrm{H}$ pylori colonisation, inflammation (Inflamm), activity, atrophy, epithelial lesions (Epit les), or intestinal metaplasia (Int met).

of $H$ pylori infection within normal household contacts, because it does not involve a genetic contribution of susceptibility to the infection (children of $H$ pylori positive subjects) or transmission via contaminated instruments (nurses or physicians). To date, however, very few studies have dealt with this topic, yielding conflicting results. Malaty and coworkers, ${ }^{3}$ studying the familial clustering of $H$ pylori infection, showed that $68 \%$ of spouses of asymptomatic infected persons with gastritis were also $H$ pylori infected, compared with only $9 \%$ of $H$ pylori negative subjects. In contrast, Perez-Perez et $a l^{5}$ and Alam et $a l^{6}$ did not find an increased prevalence of $H$ pylori infection in spouses of infected patients. The design of our study was unique because it was based on only the spouses of patients with duodenal ulcer and not on people in contact with patients affected by various pathologies (peptic ulcer, non-ulcer dyspepsia, asymptomatic gastritis, etc) which might have been sustained by different $H$ pylor strains, ${ }^{8}$ probably endowed with different degrees of virulence. In addition, the clinical relevance of $H$ pylori seropositivity in those spouses was also evaluated according to the presence or absence of dyspeptic symptoms.

The higher frequency of $H$ pylori infection that we found among the spouses of $H$ pylori positive patients with duodenal ulcer, compared with a control population matched for age, sex, geographical area of origin in Italy, and socioeconomic status, strongly supports the theory of a person to person transmission or a common source of infection, or both. This also agrees with the recent observations of Georgopolous $e t \mathrm{al}^{7}$ using the DNA fingerprint technique; indeed, in a preliminary study, they reported that nearly $50 \%$ of spouses of $H$ pylor positive patients with duodenal ulcer harboured $H$ pylori strains with the same, or similar, ribopattern as their partners. Our findings also concur with a preliminary study by Mendall et $a l^{15}$ who observed that marital status is an independent risk factor for $H$ pylori infection, and with the data of Schutze et al ${ }^{16}$ who showed that $H$ pylori infection may be transmitted by a patient's spouse. Although, generally speaking, volunteer blood donors may not be fully comparable with the general population because of self selection, we consider our blood donors sufficiently representative of the general population living in Milan because they also included peptic ulcer patients and they had a wide social class distribution. In this regard, it must be underlined that the seropositivity rate of our blood donors was quite comparable with those recently reported in the general population of Northern Italy ${ }^{9}$ and in a large group of blood donors living in the city of Bologna. ${ }^{17}$

Our findings also add more information to the problem of $H$ pylori transmission within couples, that of spouses of $H$ pylori positive duodenal ulcer patients appearing to be at an increased risk of developing a peptic ulcer; at a first glance, it would seem that such a risk is very high. Indeed, if we consider only spouses who underwent endoscopy, the prevalence of peptic ulcer was $25 \%$ (12 of 48 ); such a frequency, however, might be overestimated because most of the symptomatic spouses $(86 \%)$ agreed to undergo endoscopy compared with less than half $(38 \%)$ of those who had never suffered from dyspepsia. This is indirectly confirmed by the observation that all but one peptic ulcer and the vast majority of endoscopic gastroduodenal lesions were found in symptomatic spouses. Despite this fact, the prevalence of peptic ulcers among $H$ pylori positive spouses remains high, if we consider that in a recent study of Tibetan monks with a similar prevalence of $H$ pylori infection (75\%) but greater frequency of dyspepsia $(69 \%)$, Katelaris $e t a l^{18}$ found a $6.6 \%$ endoscopic point prevalence of peptic ulcer.

As far as the clinical relevance of $H$ pylori seropositivity in asymptomatic spouses is concerned, our study shows that the frequency of major gastroduodenal lesions (namely peptic ulcers) in $H$ pylori positive asymptomatic subjects is rather low. Indeed, only one of $22(5 \%)$ never symptomatic $H$ pylori positive spouses had peptic ulcer at endoscopy. This concurs well with that recently observed by Dooley et $a l^{19}$ and Anand $e t a l^{20}$ in asymptomatic volunteers, and suggests that the probability of peptic ulcer in the absence of chronic or recurrent dyspepsia is low.

In conclusion, our study shows that being the spouse of an $H$ pylori positive patient with duodenal ulcer increases the risk of $H$ pylori colonisation and probably of peptic ulcer disease. This provides further evidence for a person to person transmission of the infection and raises questions as to whether serological screening of cohabiting partners of $H$ pylori positive patients with duodenal ulcer may be indicated.

Part of this paper was presented at the VIIIth International Workshop of the European $H$ pylori Study Group, Edinburgh $7-9$ July 1995, and published in abstract form in Gut $1995 ; 37$ (suppl 1): A23.

1 Drumm B, Perez-Perez CI, Blaser MJ, Sherman PM. Intrafamilial clustering of Helicobacter pylori infection N Engl F Med 1990; 322: 359-63.

2 Bamford KB, Bickley J, Collins JS, et al. Helicobacter pylori: comparison of DNA fingerprints provides evidence for intrafamilial infection. Gut 1993; 34: 1348-50.

3 Malaty HM, Graham DY, Klein PD, Evans DG, Adam E, Evans DJ. Transmission of Helicobacter pylori infection. Studies in families of healthy individuals. Scand $f$ Gastroenterol 1991; 26: 927-32. 
4 Oderda G, Vaira D, Holton J, et al. Helicobacter pylori in children with peptic ulcer and their families. Dig Dis $S c i$ 1991; 36: 572-6.

5 Perez-Perez GI, Witkin SS, Decker MD, Blaser MJ Seroprevalence of Helicobacter pylori infection in couples. F Clin Microbiol 1991; 29: 642-4.

6 Alam K, Cutler A, Schubert T. Prevalence of Helicobacter pylori in spouses of infected patients. Gastroenterology 1993; 104: A31.

7 Georgopoulos S, Mentis A, Spiliadis C, Stabolos P, Tzouvelekis L, Skandalis N. Helicobacter pylori infection in spouses of duodenal ulcer patients Ribopattern comparison. Am 7 Gastroenterol 1994; 89: Ribop

8 Yoshimura HH, Evans DG, Graham DY. DNA-DNA hybridization demonstrates apparent genetic differences between Helicobacter pylori from patients with duodenal ulcer and asymptomatic gastritis. Dig Dis Sci 1993; 38: 1128-31.

9 Eurogast Study Group. Epidemiology of, and risk factors for Helicobacter pylori infection among 3194 asymptomatic subjects in 17 populations. Gut 1993; 34: 1672-6.

10 Price AB. The Sidney system: histologic division. Eur $\mathcal{f}$ Gastroenterol Hepatol 1991; 6: 209-22.

11 Parente F, Maconi G, Sangaletti O, Minguzzi M, Vago L Bianchi Porro G. Behaviour of acid secretion, gastrin release, serum pepsinogen I and gastric emptying of release, serum pepsinogen I and gastric emptying of
liquids over six months from eradication of Helicobacter liquids over six months from eradication of Helicobacter
pylori in duodenal ulcer patients. A controlled study. Gut pylori in duodenal
12 Lambert JR, Lin SK, Nicholson $\mathrm{L}$, et al. High prevalence of $\mathrm{H}$. pylori antibodies in institutionalized adults. Gastroenterology 1990; 98: A74.

13 Harris AZ, Douds A, Meurisse EV, Dennis M, Chambers S, Gould SR. Seroprevalence of Helicobacter pylori in residents of a hospital for people with severe earning difficulties. Eur $\mathcal{f}$ Gastroenterol Hepatol 1995; 7: 721-3.

14 Wilhoite S, Ferguson D, Soike D, Kalbfleisch J, Thomas E Increased prevalence of Helicobacter pylori antibodies among nurses. Arch Intern Med 1993; 153: 70-2.

15 Mendall MA, Molineaux N, Levi J, Strachan D, Northfield TC. Evidence for interpersonal transmission of H. pylori between adults. Gut 1995; 37 (suppl 1): A10.

16 Schutze K, Hentschel E, Dragosics B, Hirschl AM Helicobacter pylori reinfection with identical organisms: transmission by the patients' spouses. Gut 1995; 36 831-3.

17 Vaira $\mathrm{D}$, Miglioli $\mathrm{M}$, Mulè $\mathrm{P}$, et al. Prevalence of peptic ulcer in Helicobacter pylori positive blood donors. Gut 1994, 35: 309-12.

18 Katelaris PH, Tippet G, Brennen R, Norbu P, Farthing MJ Prevalence of Helicobacter pylori and peptic ulcer and relation to symptoms in a Tibetan refugee population in southern India. Gut 1991; 32: A556.

19 Dooley CP, Cohen H, Fitzgibbons PL, et al. Prevalence of Helicobacter pylori infection and histologic gastritis in Helicobacter pylori infection and histologic gastritis in

20 Anand BS, Malaty H, Genta RM, Graham DY. Peptic ulcer is rare in asymptomatic adults with Helicobacter pylori infection. Gut 1995; 37 (suppl 1): A10. 